\title{
Rôle des clusters dans la dynamique du développement de l'attractivité territoriale. Cas du pôle technologique El-Ghazala en Tunisie
}

\author{
Samia Haddad, Maître assistante \\ Université du 7 novembre à Carthage, Tunisie \\ Faculté des Sciences Économiques et de Gestion de Nabeul
}

\section{INTRODUCTION}

\section{Les politiques publiques visent souvent à créer les conditions de démarrage et de développement de parcs technologiques et scientifiques afin de promouvoir la dynamique de l'innovation technologique.}

Visant à promouvoir l'attractivité des territoires, les politiques économiques reposent en premier lieu sur la mise à disposition d'infrastructures publiques, susceptibles de favoriser le développement d'investissements privés. Le décideur public ambitionne alors de créer les conditions permettant de susciter la création et le développement des clusters en vue d'accroître la productivité des facteurs de production privés. Certes, les politiques publiques visent souvent à créer les conditions de démarrage et de développement de parcs technologiques et scientifiques afin de promouvoir la dynamique de l'innovation technologique. Il s'agit alors de mettre à la disposition des entreprises étrangères les ressources adéquates (infrastructures publiques, proximité de centres de formation et de recherche, etc.) pour favoriser la construction de réelles complémentarités productives entre ces structures industrielles.

Pour être efficaces, de telles politiques devraient favoriser l'implantation des entreprises certes diverses mais complémentaires. Elles mettent en place des mesures incitatives à l'implantation des entreprises étrangères sur un territoire aménagé en cluster. Ainsi, le développement des clusters s'appuie sur des dynamiques très variées, où les phénomènes spontanés et des politiques publiques volontaristes interagissent selon des formes et en suivant des séquences chronologiques très différentes d'un cas à l'autre'.
Relevant d'une démarche volontariste, le pôle technologique El-Ghazala constitue une forme de territorialisation des activités de production et d'innovation technologique dans les télécommunications. Créé en 1999, à l'initiative du ministère des Technologies de la Communication, ce parc s'étend sur un site de 60 ha. Le choix de sa localisation dans la région d'Ariana (Grand Tunis) peut être expliqué de deux manières. D'abord, l'accessibilité géographique de ce cluster par rapport à des infrastructures routières, notamment sa proximité de l'aéroport Tunis-Carthage, est une première explication sur laquelle s'appuient les décideurs publics. Ensuite, il s'agissait de concrétiser les choix industriels du gouvernement tunisien en matière de télécommunications, et notamment de favoriser l'introduction de techniques à fort potentiel de développement ${ }^{2}$.

La concentration de compétences et la disponibilité foncière semblent donc avoir été des éléments déterminants dans le choix de localisation du cluster.

Aussi, ce choix s'est porté sur le site, propriété du ministère des Technologies de la Communication, dans lequel étaient installées, depuis les années 1980, l'École Supérieure des Postes et des Télécommunications de Tunis (ESPTT) ${ }^{3}$ et l'imprimerie de la poste. La concentration de compétences et la disponibilité foncière semblent donc avoir été des éléments déterminants dans le choix de localisation du cluster.

Les pouvoirs publics jouent donc un rôle prépondérant à tous les niveaux de la construction de ce cluster, et cela, dans un contexte politique très centralisé ${ }^{4}$. Ainsi, leur volonté de 
procéder à un ancrage territorial d'activités de production industrielle en matière de techniques et services de communication s'est traduite par l'engagement du ministère des Communications dans la gestion du projet d'aménagement du territoire $^{5}$. En effet, le ministère a financé entièrement le site, du foncier aux bâtiments. Il s'est aussi doté de moyens de fonctionnement (personnel, réseaux de communication, etc.) et de financement à partir de son propre budget. Dans ces conditions, le pôle technologique ElGhazala est non seulement devenu un lieu où se croisent des compétences scientifiques et des acteurs industriels, mais aussi un espace au service de l'attractivité territoriale.

\section{MÉTHODOLOGIE}

Dans le cadre de ce travail de recherche, les données mobilisées sont issues d'une enquête, réalisée en septembre 2009, auprès des firmes multinationales installées dans le cluster d'ElGhazala. L'objectif de l'enquête consiste à identifier les facteurs d'attractivité des entreprises étrangères, spécialisées dans le secteur de TIC. Le questionnaire a été conçu sur la base d'une large revue de littérature théorique ${ }^{7}$ qui a permis d'apprécier les facteurs déterminants de la localisation des firmes dans le parc technologique El-Ghazala. L'enquête s'est déroulée par des entretiens directs auprès des responsables des entreprises étrangères. L'échantillon est composé de 8 firmes multinationales implantées dans le pôle technologique. Environ $60 \%$ de ces entreprises sont d'origine euro-
Partant de ce constat, le présent article propose d'analyser le rôle que peut jouer le cluster ElGhazala dans la dynamique du renforcement de l'attractivité des entreprises étrangères et donc dans leur choix d'implantation. L'enquête menée auprès des 8 firmes multinationales ${ }^{6}$ installées dans ce parc nous permettra d'identifier les principaux facteurs d'attractivité spécifiques à ce pôle technologique, spécialisé dans l'ingénierie informatique et de télécommunication. Ainsi, nous présentons la source de données utilisées pour étudier les facteurs d'attractivité du pôle technologique El-Ghazala, spécialisé dans le secteur des TIC. Puis, nous exposons les résultats relatifs à l'identification de ces facteurs.

péenne (principalement la France, l'Italie, l'Allemagne et la Finlande).

Étant des entreprises totalement exportatrices, elles procèdent à un investissement direct étranger de type vertical. Leur investissement est principalement réalisé dans deux domaines d'activités : le premier s'articule autour des services réseaux à valeur ajoutée (produit de développement de solutions); le deuxième porte sur des activités de conseil et de formation. Leurs produits et services sont destinés aux marchés étrangers, notamment au marché africain. Les informations qualitatives obtenues ont permis d'identifier les facteurs d'attractivité des entreprises étrangères retenues pour les fins de cette étude.

\section{IDENTIFICATION DES PRINCIPAUX FACTEURS D’ATTRACTIVITÉ DU PARC TECHNOLOGIQUE EL-GHAZALA}

Les résultats de l'enquête font ressortir cinq groupes relatifs aux facteurs déterminants de la localisation des firmes multinationales dans le parc. Premièrement, les facteurs inhérents aux externalités pécuniaires sont très importants dans la dynamique de localisation des firmes. En effet, ils renvoient en dehors de la demande locale à l'ensemble des facteurs qui contribuent à réduire les coûts de production à savoir : marché potentiel important localement, main- d'œuvre qualifiée, proximité aux sous-traitants et fournisseurs, infrastructures spécifiques à l'entreprise et infrastructures routières. Deuxièmement, les facteurs liés aux externalités de réseaux dont bénéficie le site El-Ghazala. Il s'agit d'un lieu économiquement privilégié où les firmes pourront bénéficier des rapprochements et des interactions avec les acteurs situés sur ce même site. Ainsi, quatre variables sont incluses dans ces facteurs (réseau 
d'affaires importants, proximité aux écoles et/ou universités, proximité de technopoles, réseaux TIC). Troisièmement, les facteurs liés aux aménités urbaines qu'offre le territoire d'accueil en matière de qualité de vie.

Quatrièmement, les facteurs personnels d'attachement au territoire des dirigeants de la firme auraient pu dicter la décision d'implantation au sein du site El Ghazala (appartenance au territoire, réseau familial local, contact avec les élus locaux et climat politique et social).

Enfin, les aides et subventions à la localisation constituent des facteurs non négligeables. Bien que le pôle ne bénéficie pas des incitations propres aux trois zones d'encouragement au développement régional prévu par le code des investissements, les entreprises qui y sont implantées bénéficient d'une exonération d'impôts pendant les cinq premières années de leur activité $^{8}$. Cette faible incitation peut constituer une menace de délocalisation de ces firmes, car l'État offre des subventions aux entreprises en fonction du type de zone d'encouragement au développement régional (premier groupe, second groupe ou zone prioritaire). On estime toutefois que l'impact des subventions pourrait dépendre de l'importance des externalités de réseau qu'offre la technopole qui s'attend à ce que les décisions de localisation puissent être dictées par d'autres considérations. On pense, entre autres, aux externalités de réseaux et aux externalités pécuniaires.

\section{À part les infrastructures routières, les autres facteurs d'attractivité territoriale inhérents aux externalités pécuniaires ne semblent pas être négligeables dans les décisions d'implantation des firmes. En effet, la main-d'œuvre qualifiée est le facteur le plus attractif, suivi de la proximité aux sous-traitants.}

À part les infrastructures routières, les autres facteurs d'attractivité territoriale inhérents aux externalités pécuniaires ne semblent pas être négligeables dans les décisions d'implantation des firmes. En effet, la main-d'œuvre qualifiée est le facteur le plus attractif, suivi de la proximité aux sous-traitants, tel qu'illustré au graphique 1.

\section{Graphique 1 : Impact des facteurs liés aux externalités pécuniaires sur la localisation des entreprises étrangères dans le cluster}

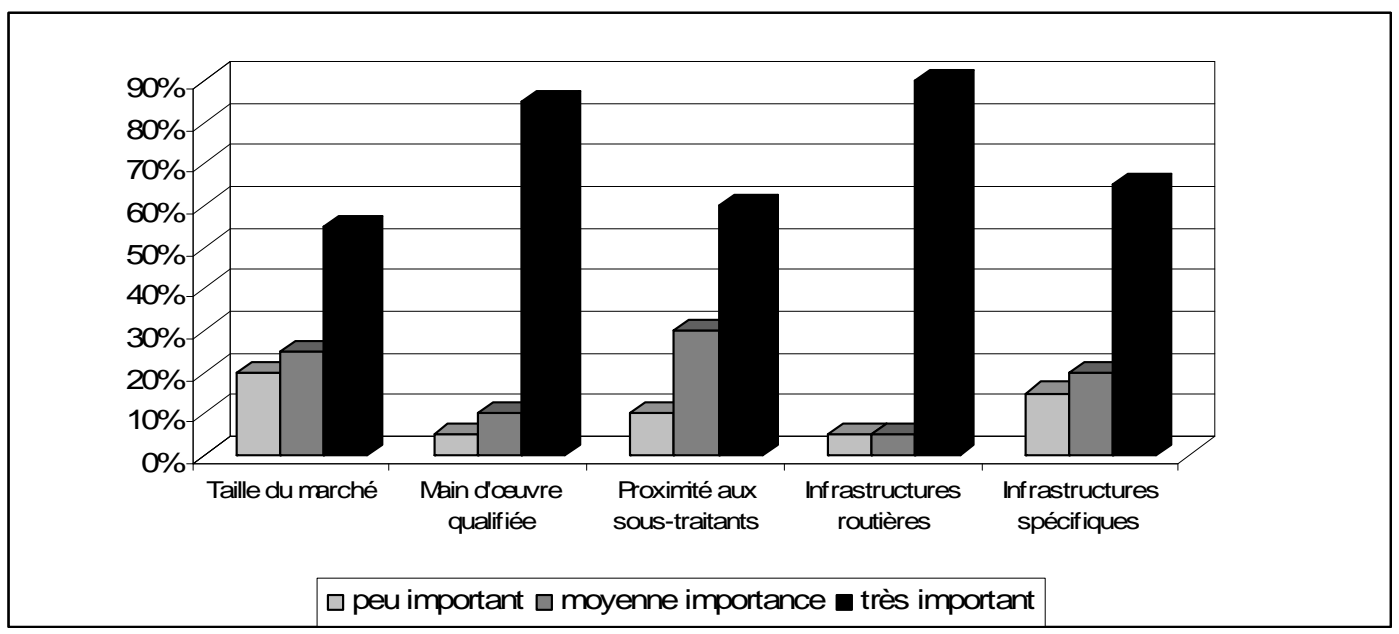

En outre, la taille du marché constitue un facteur déterminant de localisation pour $55 \%$ des entreprises étrangères enquêtées. Dans ce cadre, le cluster El-Ghazala se définit par ses performances à l'exportation des produits et services liés aux techniques de télécommunication, principalement à destination du marché régional et arabe (Maroc, Afrique francophone, Syrie, Jordanie), mais également des marchés américain et européen (France, Grande-Bretagne). 
Les facteurs liés aux aménités culturelles et de loisirs et d'attachement personnels au territoire ne semblent pas être très déterminants dans les décisions d'implantation (Graphique 2). En effet, les responsables des entreprises étrangères, installées au parc El-Ghazala, considèrent que leur appartenance au territoire ne constitue pas un facteur très important de choix d'implantation. Toutefois, ils estiment que le contact avec les élus locaux et le climat politique et social est très décisif dans le choix d'implantation de $85 \%$ des firmes multinationales.

Graphique 2 : Importance des facteurs liés aux aménités culturelles et d'attachement personnel au territoire dans le choix d'implantation

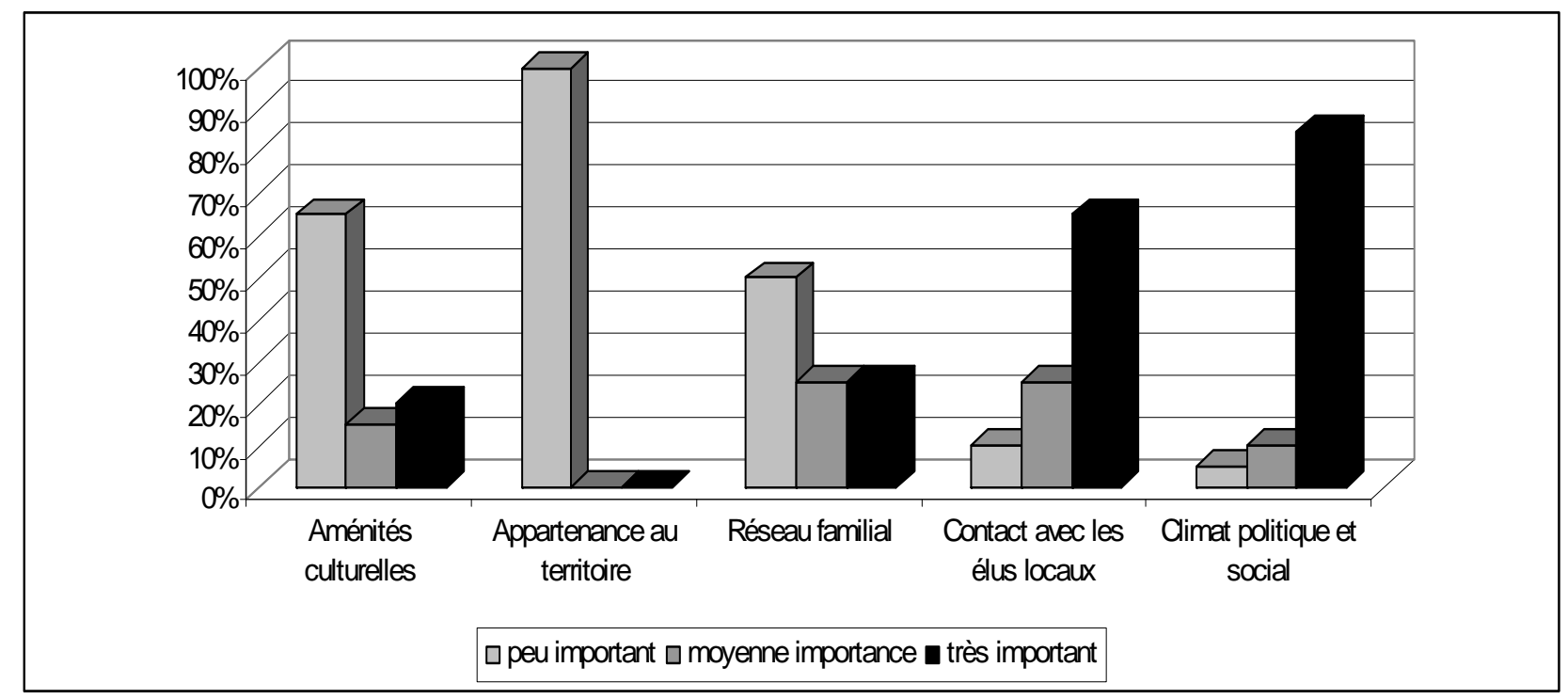

La firme multinationale Alcatel offre aux entreprises locales sa plate-forme équipement, afin qu'elles puissent assurer le développement des services à valeur ajoutée (SMS ou MMS).

Les facteurs liés aux externalités de réseau semblent plus déterminants dans le choix d'implantation par rapport aux autres facteurs (Graphique 3). La plupart des firmes accordent une importance moyenne aux facteurs leur permettant de bénéficier des rapprochements et des interactions avec les acteurs situés sur ce même site (entreprises, universités, administration, réseau TIC). D'ailleurs, plusieurs entreprises affirment avoir des synergies avec les entreprises de leurs entourages. À titre d'illustration, la firme multinationale Alcatel offre aux entreprises locales sa plate-forme équipe-ment, afin qu'elles puissent assurer le dévelop-pement des services à valeur ajoutée (SMS ou MMS). Le reste, soit $25 \%$, développe des synergies avec la totalité des entreprises.
Les synergies avec les universités et la proximité à la technopole sont aussi des facteurs très attractifs pour des entreprises. Les synergies portent essentiellement sur le développement des contrats de coopération avec les universités du pôle, en offrant notamment des stages aux étudiants dans le cadre de leur projet de fin d'études ou de master. Les entreprises participent aussi à l'encadrement de thèse au sein des universités. Ces synergies répondent donc plus aux besoins en main-d'œuvre qualifiée des firmes multinationales (en sélectionnant les meilleurs ingénieurs à recruter) qu'au développement des externalités de connaissance.

Les synergies portent essentiellement sur le développement des contrats de coopération avec les universités du pôle, en offrant notamment des stages aux étudiants dans le cadre de leur projet de fin d'études ou de master. 


\section{Graphique 3 : Impact des facteurs inhérents aux externalités de réseaux dans le choix d'implantation des firmes étrangères}

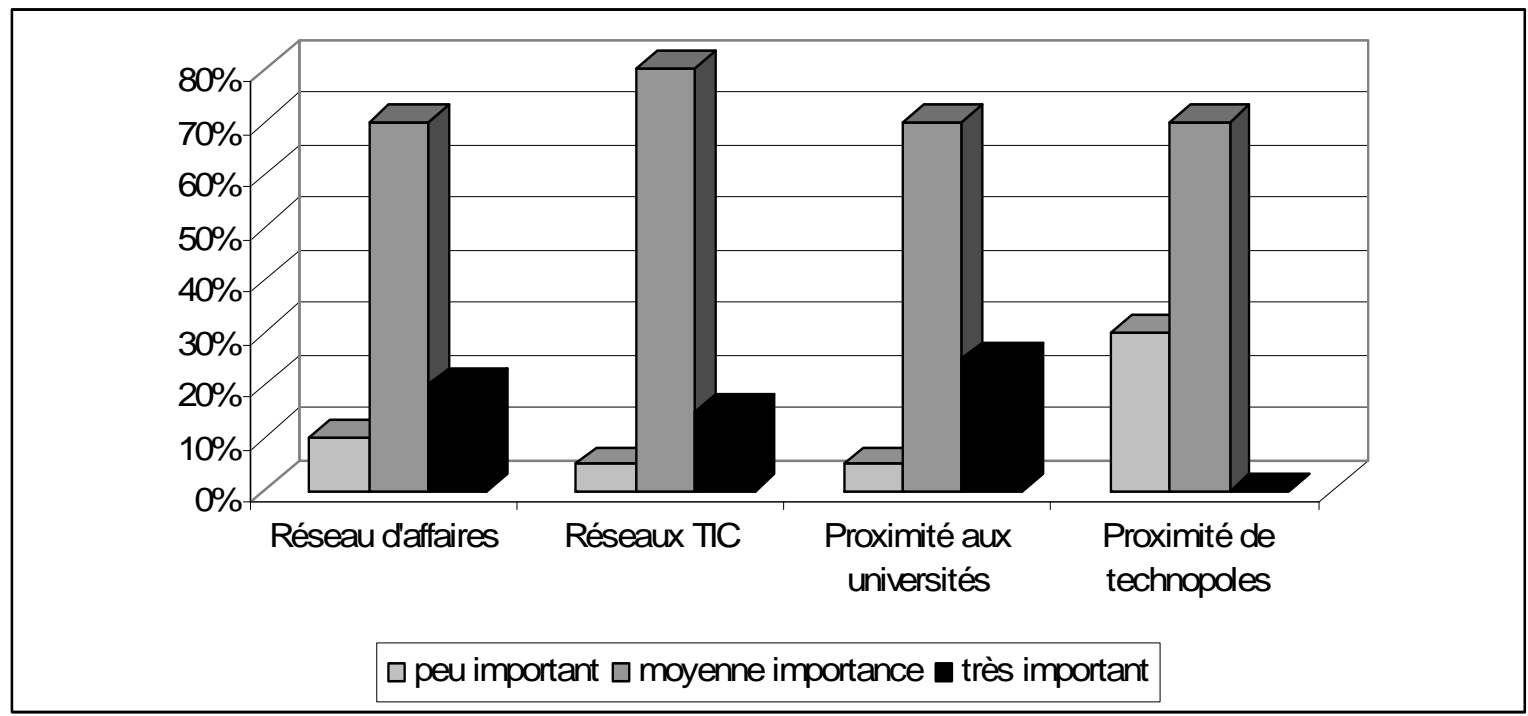

Tel que présenté au graphique 4 , les aides ont un réel effet incitatif sur la localisation des entreprises. En effet, pour la moitié des entreprises étudiées, les aides ont contribué à leur décision d'implantation au sien du site El Ghazala. Les subventions constituent le facteur le moins attractif dans leur choix de localisation. D'ailleurs, aucune firme n'a cherché à délocaliser vers une autre région leur offrant des subventions intéressantes.

\section{Graphique 4 : Contribution des incitations fiscales dans la décision d'implantation}

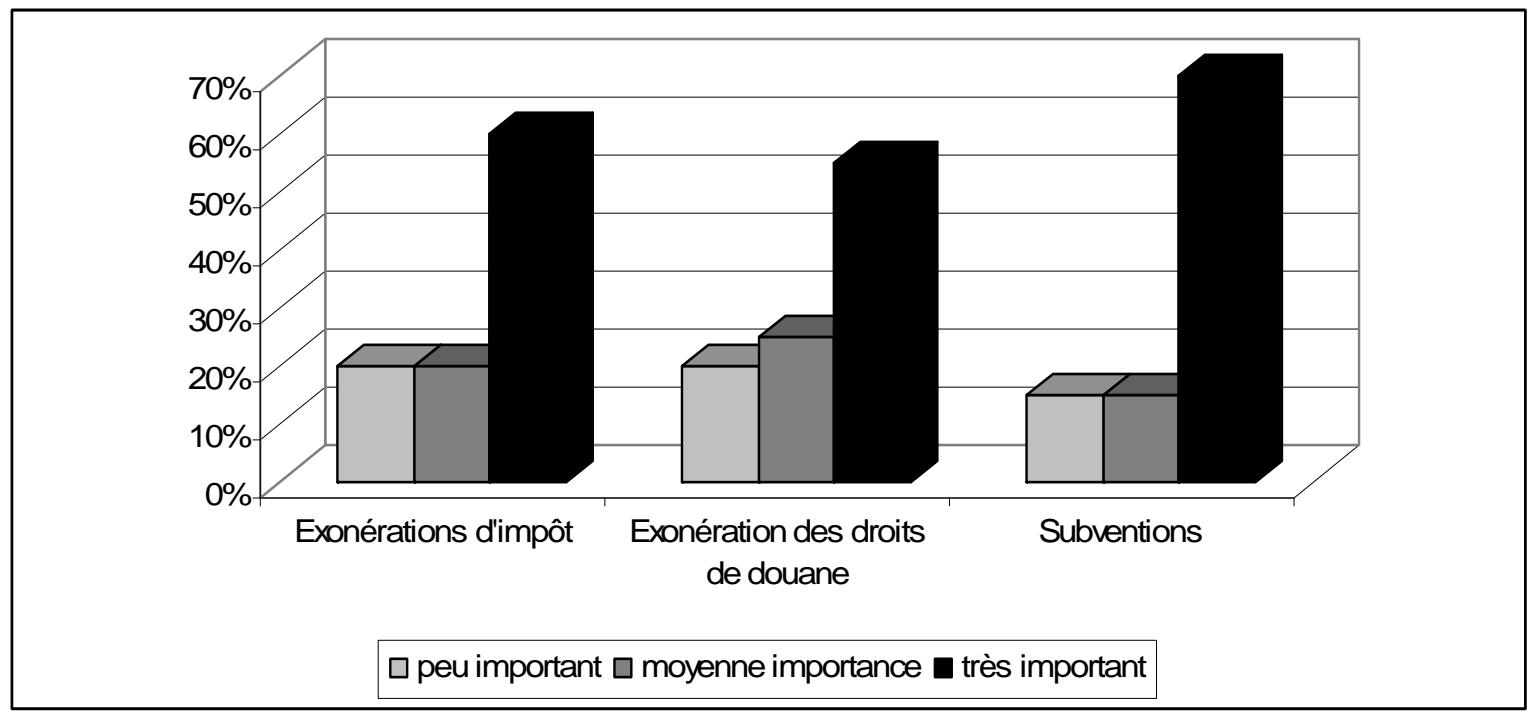

Le processus de création et de développement du parc technologique El-Ghazala s'appuie donc essentiellement sur des économies d'agglo- mération (réseau d'affaire important, proximité aux écoles et aux universités, proximité aux 
sous-traitants et fournisseurs, main-d'œuvre

\section{CONCLUSION}

\section{La politique des clusters consiste à attirer des groupes industriels, considérés comme les vecteurs essentiels des transferts de compétences, dans des secteurs d'activités pointus.}

Lancée en Tunisie en 1999, la politique des clusters consiste à attirer des groupes industriels, considérés comme les vecteurs essentiels des transferts de compétences, dans des secteurs d'activités pointus. Elle permet aussi d'intensifier les fertilisations croisées entre les structures de recherche, les institutions d'enseignement et l'industrie et de favoriser l'émergence de projets innovants, créateurs de valeur ajoutée et d'emploi.

C'est bien dans cette perspective que le processus de création du pôle El-Ghazala, dédié aux techniques de l'information et de la communication, a été impulsé. Certes, la création de ce cluster a permis de favoriser l'attraction des entreprises d'origines extérieures. Il offre un environnement favorable à l'accueil d'entreprises spécialisées (bassin d'emploi offrant une main-d'œuvre qualifiée, compétences scientifiques et techniques de rayonnement international, infrastructures et circuits de financement, proximité aux soustraitants et fournisseurs, etc.). Il sert aussi de plate-forme à l'émergence et le développement de projets innovants.

Enfin, le cluster crée un effet d'image, fondé sur l'existence et la promotion d'une offre territoriale différenciée qui peut susciter un intérêt spontané auprès des acteurs extérieurs. À son tour, l'afflux des entreprises extérieures peut renforcer l'attractivité du cluster à travers des effets de dynamique d'agglomération. En effet, elles contribuent à intégrer le pôle technologique dans des réseaux internationaux de partenariats et de circulation des savoirs, des échanges de financement et des compétences, mais aussi elles interviennent dans la diffusion des savoir-faire et des capacités d'innovations dans des secteurs d'activités stratégiques. Ce qui qualifiée).

justifie ainsi le lancement de politiques d'attractivités actives destinées à promouvoir le cluster auprès des partenaires étrangers potentiels.

Toutefois, des réserves peuvent être prononcées concernant les politiques d'attractivité des clusters. En effet, beaucoup d'investissements étrangers sont réalisés dans le seul but, par exemple, d'accéder à un avantage comparatif en main-d'œuvre qualifiée sans pour autant générer des effets d'agglomération positives (faible voire absence de réseaux de collaboration entre firmes étrangères - instituts de recherche universités et les firmes domestiques). Dans ce contexte, et dans leurs politiques de promotion et développement des clusters, les décideurs politiques tunisiens sont conduits à articuler fortement les politiques de développement endogène des clusters en permettant aux entreprises étrangères de renforcer les économies d'échelle dans la création de connaissance. Aussi, leur politique de développement des clusters devraient promouvoir davantage les capacités scientifiques et technologiques des acteurs locaux, porteurs de projets innovants, en rapprochant les instituts de recherche, les universités et les grandes firmes technologiques. De telles politiques permettraient au cluster de devenir un lieu d'accueil privilégié des activités innovantes et de $R \& D$ des entreprises étrangères.

\section{Leur politique de développement des clusters devraient promouvoir davantage les capacités scientifiques et technologiques des acteurs locaux, porteurs de projets innovants, en rapprochant les instituts de recherche, les universités et les grandes firmes technologiques.}

\section{BIBLIOGRAPHIE}

${ }^{1}$ Hatem, F., 2007. «Le rôle des clusters dans les politiques d'attractivité», L'attractivité des territoires: Regards croisés, Actes des Séminaires, pp.19-22.

${ }^{2}$ Haddad, S. et Poncet, Ch., 2008. «Clusters et dynamique de l'innovation dans les télécommunications sur le site d'Elgazala en Tunisie», 
Laperche, B., (Ed.), L'innovation pour le développement. Enjeux globaux et opportunités locales. Édition Karthala, Paris, pp. 93-115.

${ }^{3} \mathrm{~A}$ la suite d'une restructuration de l'ESPTT en 1998, deux écoles ont été créées: il s'agit de l'Institut Supérieur des Études Technologiques en Communi-cations (ISET'Com) et de l'école supérieure des communications (Sup'Com).

${ }^{4}$ Fekih, B., Haddad, S., (2009). «Les déterminants du dynamisme technologique dans les clusters : cas du pôle technologique El-Ghazala», Les cahiers de l'Association Tiers-Monde, $\mathrm{n}^{\circ} 24$, pp.157-174

${ }^{5}$ La gestion du parc a été confiée à un établissement public à caractère non administratif, appelé "Pôle ElGhazala des technologies de la communication », sous la tutelle du ministère des Technologies de la communication.

${ }^{6}$ Il s'agit de : Alcatel, Ericsson, ST Microelectronics, Huawei Technologies, StoneSoft, Actielec Technologies, IGE-XAO et Kromberg \& Schubert.

${ }^{7}$ Larribeau, S., Oukarfi, S., (2004). «Efficacité des aides à la localisation: un essai de mesure sur régions françaises». Journées de la micro-économie, Tunisie, Hammamet, Mai.

${ }^{8}$ La Tunisie a modifié le décret $n^{\circ} 99-483$ du 1er mars 1999 portant délimitation des zones d'encouragement au développement régional. Le nouveau décret $\mathrm{n}^{\circ}$ 2008-387 du 11 février 2008 prévoit une troisième zone d'encouragement prioritaire dans les secteurs de l'industrie, de l'artisanat et de quelques activités de service. 
2010

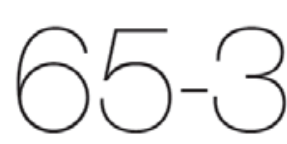

AUTOMNE

FALL

\section{REVUE TRIMESTRIELLE}

RELATIONS INDUSTRIELLES

Revue trimestrielle bilingue publiée depuis 1945 par le Département des relations industrielles de l'Université Laval

\section{ARTICLES}

La coordination patronale face à

I'ouverture des marchés : I'industrie suisse des machines depuis les années 1990

FRÉDÉRIC WIDMER

Le maintien dans I'emploi des travailleurs âgés dans trois entreprises belges

FRANÇOISE BERTRAND, CECILE LEMAIRE, MARIE BARBIER ET ISABELLE HANSEZ

La formation en organisation : mise en perspective des approches psychosociologiques et ergonomiques

DANIEL FAULX ET LUCIE PETIT

Une analyse longitudinale (1975-2005)

de l'évolution de la profession ressources humaines

VICTOR Y. HAINES III, SOPHIE BROUILLARD ET NATHALIE CADIEUX

RI/IR EN LIGNE

RI/IR est disponible en ligne sur le site Erudit: www.erudit.org/revue/ri

Pour abonnement à la version numérique, contacter Érudit.

Pour consulter les sommaires des numéros publiés, les résumés d'articles ou pour vous abonner à la version papier, visitez notre site Internet: www. riir.ulaval.ca

\section{INDUSTRIAL RELATIONS QUARTERLY REVIEW}

A bilingual quarterly published since 1945 by the Industrial Relations Department, Université Laval

\section{ARTICLES}

The Regulation of Paid Care Workers' Wages and Conditions in the Non-Profit Sector: A Toronto Case Study SARA CHARLESWORTH

Employment Relations in Chile: Evidence of HRM Practices JENNY K. RODRIGUEZ

Pay Satisfaction, Job Satisfaction and Turnover Intent

PARBUDYAL SINGH AND NATASHA LONCAR

RI/IR ON LINE

$\mathrm{RI} / \mathrm{R}$ is available on line on Erudit website at: www.erudit.org/revue/ri

To subscribe to the online version, please contact Erudit.

Visit our website for contents listings, abstracts, or to subscribe to the print edition: www. riir.ulaval.ca

\section{RELATIONS INDUSTRIELLES INDUSTRIAL RELATIONS}

Pavillon J.-A.-DeSève, 1025, avenue des Sciences-Humaines Bureau 3129, Université Laval, Québec (QC), Canada, G1V OA6 TELEPHONE : (418) 656-2468 TELECOPIEUR / FAX : (418) 656-7688 COURRIEL / E-MAIL : relat.ind@rlt.ulaval.ca www.riir.ulaval.ca 